\title{
High-flux capillary based XUV source via the direct engineering of a laser induced ionization profile
}

\author{
Patrick N. Anderson'1, Thomas J. Butcher ${ }^{1}$, Peter Horak ${ }^{1}$, Jeremy G. Frey ${ }^{2}$, William S. Brocklesby ${ }^{1}$ \\ 1. Optoelectronics Research Centre, University of Southampton, Southampton SO17 1BJ, United Kingdom \\ 2. School of Chemistry, University of Southampton, Southampton SO17 1BJ, United Kingdom
}

High harmonic generation (HHG) has proven to be a fascinating and incredibly useful nonlinear optical phenomenon and has led to the realization of tabletop sources of coherent extreme ultraviolet (XUV) radiation. Capillary based geometries in particular have attracted a great deal of attention due to the lengthy regions over which HHG can occur and the potential to phase match the HHG process leading to a large increase in XUV flux [1]. Until now reabsorption of XUV radiation has been a major problem when designing high-flux sources, in both capillary and other geometries. In this work we have used computational modelling to optimize our original capillary design in an effort to minimize the absorption of the generated XUV. Subsequent experimental work has shown a flux increase of almost 50 times over our existing capillary-based source, regardless of the maximum gas pressure.

Our three dimensional model [2] is based on a multimode-generalized nonlinear Schrödinger equation and includes effects such nonlinear mode mixing and ionization induced nonlinear effects. Using the model we adjusted both the capillary length and gas density profile with two aims: to maximize the radially integrated ionization fraction at the capillary exit coupled with a rapid decrease in gas pressure. This has the effect of increasing not only the XUV flux but also pushes the cut-off limit of the generated harmonics to higher energies as more ionization is a result of higher pump-pulse intensity. The XUV range that we can generate within presently is restricted to between $18-40 \mathrm{~nm}$ by the Cooper minimum and argon absorption respectively. For our $75 \mu \mathrm{m}$ radius capillary we found the optimum design to be $45 \mathrm{~mm}$ long with gas inlets $5 \mathrm{~mm}$ and $3 \mathrm{~mm}$ from the input and output respectively. The theoretical gas density and integrated ionization profiles are given in Figures $1(\mathrm{a})$ and $1(\mathrm{~b})$.
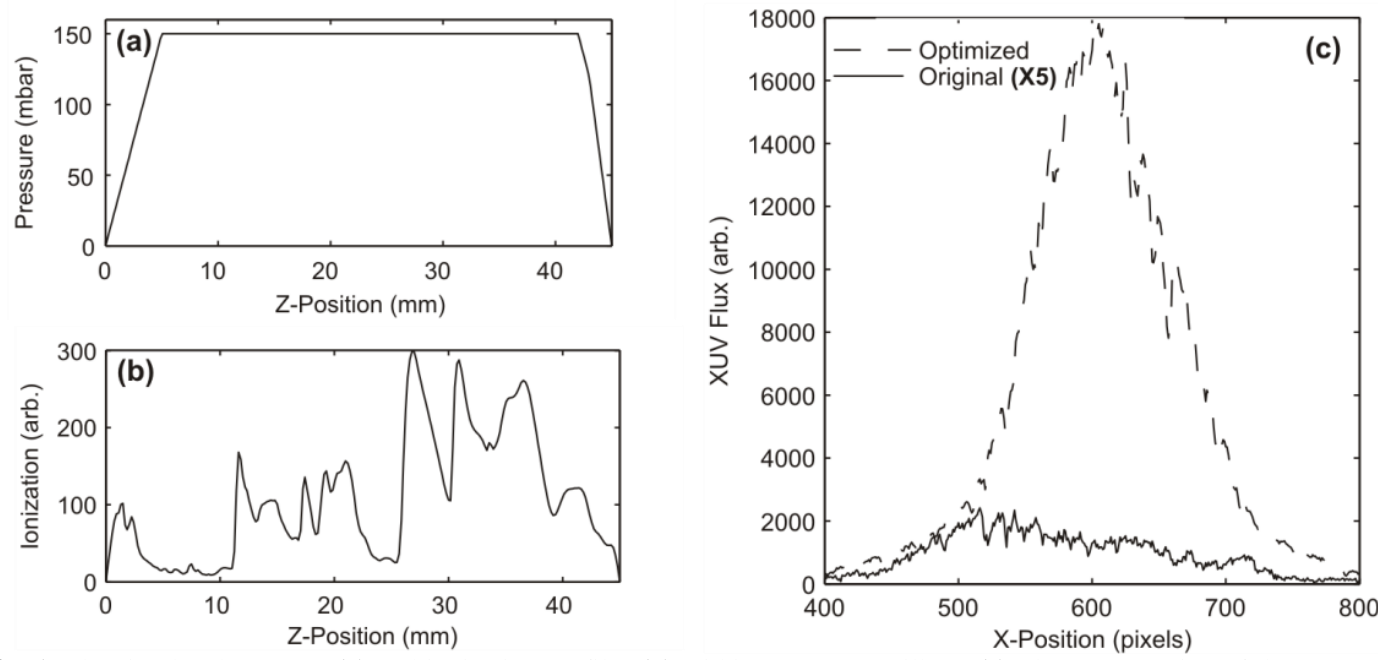

Fig. 1. The simulated pressure (a) and ionization profiles (b) within our new capillary. (c) The cross-section of the XUV beam measured at the output of our optimized (dotted line) and original (solid line) capillary based sources for a peak pressure of 150 mbar. Note that the cross-section data for the original source has been multiplied by a factor of five.

Based on this theoretical work several of these new capillaries were manufactured and integrated into our existing apparatus [2]. The output XUV flux was measured on an X-ray CCD camera situated behind an aluminium filter. Figure 1(c) shows the comparison between the capillary optimized for a high integrated ionization and low reabsorption (dotted line) with the original $70 \mathrm{~mm}$ capillary design (solid line). This shows an increase in the XUV flux of almost 50 times when compared to a non-optimized source and we have observed similar increases throughout our experimental pressure range of 0-200 mbar.

We have used our theoretical model to dramatically increase the output XUV flux of a capillary based high harmonic source by reducing the reabsorption of the generated XUV whilst simultaneously increasing the final intensity and flux of the pump laser at the capillary exit.

\section{References}

[1] A. Rundquist, C. Durfee, Z. Chang, C. Herne, S. Backus, M. Murnane and H. Kapteyn, "Phase-Matched Generation of Coherent Soft XRays," Science 280, 1412 (1998)

[2] R. T. Chapman, T. J. Butcher, P. Horak, F. Poletti, J. G. Frey, W. S. Brocklesby, "Modal effects on pump-pulse propagation in an Arfilled capillary" Optics Express 18, 13279 (2010) 